\title{
Web 2.0 gestützte Blended Learning Szenarien als kritische Masse Systeme
}

\author{
Christian Lebr \\ Fachbereich Wirtschaftswissenschaft, Freie Universität Berlin
}

\section{Einleitung und Hintergrund}

Die Umstellung auf Bachelor/Master Studiengänge und die im Bologna-Prozess geforderte Kompetenzorientierung der Lehre an europäischen Hochschulen bringen neue Anforderungen an die Hochschullehre mit sich, da sie einen grundlegenden Wandel der Zielsetzungen universitärer Lehre weg von einer rein wissenschaftlichen Ausbildung hin zu einer berufsorientierten Kompetenzvermittlung implizieren (BMBF 2009, S. 1). Dies erfordert neue Lehr- und Lernformen, die diesen Zielsetzungen Rechnung tragen.

Unter dem Begriff „Kompetenz“ verstehen das BMBF und Klieme (2007, S. 72), in Anlehnung an Weinert (2001, S. 27f.), „die bei Individuen verfügbaren oder von ihnen erlernbaren kognitiven Fähigkeiten und Fertigkeiten, bestimmte Probleme zu lösen sowie die damit verbundenen motivationalen, volitionalen und sozialen Bereitschaften und Fähigkeiten, die Problemlösungen in variablen Situationen erfolgreich und verantwortungsvoll nutzen zu können.“

Um diesen neuen Anforderungen gerecht $\mathrm{zu}$ werden, erweist sich das didaktische Modell des handlungsorientierten Unterrichts als ein vielversprechender Ansatz, der den Lernenden den aktiven Umgang mit den Inhalten und Gegenständen der Lehrveranstaltungen ermöglichen kann. Dabei soll durch die Erstellung konkreter Handlungsprodukte, mit denen sich die Studierenden identifizieren und kritisch auseinandersetzen können, eine praxisnahe Auseinandersetzung mit den Inhalten der Lehrveranstaltungen erreicht und dadurch kognitive Fähigkeiten und Fertigkeiten erlangt werden, welche die Problemlösekompetenz der Lernenden nachhaltig fördern (Wopp 1986, S. 600; Gudjons 2008, S. 144).

In diesem Zusammenhang scheint der Einsatz von Web 2.0-Anwendungen in der Lehre einen vielversprechenden Mehrwert bieten zu können, der einen Beitrag dazu leisten kann, die geforderte Kompetenzorientierung der Lehre entscheidend zu unterstützen. Im weiteren Verlauf des Textes soll nun die Frage thematisiert werden, welchen Mehrwert insbesondere contentorientierte Web 2.0Anwendungen wie Wikis aber auch Web 2.0-Anwendungen im Allgemeinen in der 
Hochschullehre entwickeln können und was zu beachten ist, um diesen Mehrwert zu generieren.

\section{Die Bedeutung der kritischen Masse in Web 2.0 gestützten Lehrveranstaltungen}

\subsection{Die kritische Masse im Web 2.0}

Um den Mehrwert von Web 2.0-Anwendungen für die Lehre verstehen und daraus Handlungsanweisungen für die Konzeption von entsprechenden Lernszenarien entwickeln zu können, sollte man sich zunächst vergegenwärtigen nach welchen Prinzipien Web 2.0-Anwendungen im Allgemeinen funktionieren und welche Rückschlüsse sich hieraus für den Einsatz in der Lehre ergeben.

Der Begriff Web 2.0 wurde erstmals von Eric Knorr $(2003$, S. 2) aufgegriffen und maßgeblich durch Tim O'Reilly (2005, S. 1ff.) in seinem Artikel „What is Web 2.0?“ geprägt. O'Reilly $(2006$, S. 1) definiert Web 2.0 als “... business revolution in the computer industry caused by the move to the internet as platform, and an attempt to understand the rules for success on that new platform. Chief among those rules is this: Build applications that harness network effects to get better the more people use them. This is what I've elsewhere called harnessing collective intelligence."

Der Begriff des Netzwerkeffektes, den O'Reilly in seiner Definition aufgreift, bezeichnet dabei den Effekt, der auftritt, sobald ein Nachfrager ein Gut höher bewertet, wenn es mit Gütern anderer Nachfrager kompatibel ist und die Güter in einer komplementären Beziehung stehen (Swann 2002, S. 417 ff.; Katz und Shapiro 1994, S. 94ff.). In der Literatur wird zwischen direkten und indirekten Netzeffekten unterschieden (Clements 2004, S. 633 ff.; Katz und Shapiro 1994, S. 95ff.; Lim et al. 2003, S. 538 ).

An dieser Stelle sollen insbesondere direkte Netzeffekte im Fokus der Betrachtung stehen, da sie, wie im Folgenden gezeigt wird, einen entscheidenden Erklärungsbeitrag für die Nutzengenerierung im Web 2.0 bieten. Bei Gütern mit ausschließlich direktem Netzeffekt handelt es sich um Güter, die über einen rein derivativen, also abgeleiteten Produktnutzen verfügen und zudem im Rahmen einer Systemtechnologie eingesetzt werden. Daher werden diese Güter auch als Systemgüter bezeichnet (Weiber 1992, S. 18). Damit ein Nachfrager aus einem Systemgut einen Nutzen ziehen kann, muss mindestens ein weiteres gleichartiges Systemgut bei einem anderen Nachfrager vorhanden sein, mit dem eine Interaktionsbeziehung eingegangen werden kann. Der Aspekt der Interaktionsbeziehung ist somit der für Systemgüter nutzenstiftende zentrale Faktor. Je mehr Nachfrager Systemgüter im Rahmen der gleichen Systemtechnologie nutzen, umso höher wird der derivative Nutzen, den ein einzelner Nachfrager aus seinem Systemgut, das im Rahmen eben dieser Systemtechnologie eingesetzt wird, ziehen kann. 
Ist demnach die Größe der Nutzerbasis, hier spricht man von der installierten Basis, zu klein, ist der für den einzelnen Anwender auf Dauer erzielbare Nutzen möglicherweise zu gering und er wird die Teilnahme an der Systemtechnologie einstellen.

Der gegenteilige Fall tritt ein, wenn die installierte Basis eine bestimmte Größe überschreitet, sodass der derivative Nutzen, der von den einzelnen Anwendern erzielt werden kann, ihre Erwartung erfüllt und sie die Nutzung der Systemtechnologie beibehalten und sogar weitere Anwender angezogen werden, die ihrerseits zum einen den eigenen Nutzen aus der Systemtechnologie ziehen und andererseits den derivativen Nutzen der übrigen Anwender weiter erhöhen. Bei Systemgütern existiert demnach ein Rückkopplungseffekt, der auch den Nutzen von bereits an das System angeschlossenen Anwendern durch neu hinzukommende Anwender beeinflusst (Weiber 1992, S. 133). Die Mindestgröße der installierten Basis, beziehungsweise die Mindestanzahl an teilnehmenden Anwendern, ab der Systemgüter einen ausreichenden Nutzen für eine langfristige Nutzung erzielen, wird kritische Masse genannt (Rogers 1990). Daher bezeichnet man die Systemtechnologien, die den benötigten Rahmen für diese Systemgüter bieten, als kritische Masse-Systeme.

Folgt man O'Reillys (2006, S. 1) Definition, sind Web 2.0-Anwendungen "applications that harness network effects to get better the more people use them." Somit besitzen Web 2.0-Anwendungen in der Regel den Charakter von kritischen Masse-Systemen. Zum Verständnis der Nutzengenerierung in Web 2.0Anwendungen bietet also die Diffusion in kritische Masse-Systemen einen entscheidenden Beitrag (Liehr 2005, S. 23; Kroeber-Riel, Weinberg et al. 2009, S. 672).

\subsection{Diffusions- und Adoptionsprozesse im Web 2.0}

Die Diffusionstheorie untersucht die Verbreitung und Akzeptanz von Innovationen in sozialen Systemen (Rogers 2003, S. 5f.). Ausgehend von der Adoption einer Innovation, also der Übernahme dieser Innovation durch einen individuellen Nachfrager, werden die verschiedenen individuellen Adoptionsprozesse aller Nachfrager zu einem gemeinsamen Diffusionsprozess aggregiert, der so eine Gesamtbetrachtung der Ausbreitung einer Innovation in einem sozialen System ermöglicht (Rogers 2003, S. 12).

Während bei der klassischen Diffusionstheorie, die sich mit der Adoption und Diffusion von Singulärgütern befasst, der Prozess der Adoption eines Gutes mit der Übernahme, also dem Kaufakt dieses Gutes durch einen Nachfrager, abgeschlossen ist, muss bei der Adoption eines kritische Masse-Systems eine Erweiterung des Adoptionsprozesses berücksichtigt werden. Dieser erweiterte Adoptionsprozess ergibt sich daraus, dass bei kritische Masse-Systemen der Nutzen erst durch die Interaktion mit anderen Nutzern entsteht. Dadurch ist die Adoption eines kritische Masse-Systems nicht wie bei Singulärgütern mit dem Zeitpunkt des Kaufaktes abgeschlossen, sondern ergibt sich erst aus der eigentlichen Nutzung des Systems. Neben dem Kaufakt ist also bei kritische Masse-Systemen der An- 
schluss- und im besonderen Maße der Nutzungsakt der für die Adoption entscheidende nutzenstiftende Faktor.

Für die kritische Masse bedeuten diese Überlegungen, dass ab dem Punkt an dem die Nutzungsintensität im Gesamtsystem ausreichend groß wird, sich die Adoptionsrate selbst erhält und somit der Diffusionsprozess von einer Phase der Instabilität in eine Phase der Stabilität wechselt (Rogers 2003, S. 343). Überschreitet ein kritisches Masse-System diesen Punkt, verläuft die weitere Diffusion des Systems mit einer hohen Eigendynamik und in aller Regel erfolgreich.

Aus diesen Überlegungen lässt sich die These ableiten, dass Web 2.0Anwendungen in der Lehre vor allem dann einen Mehrwert generieren können, wenn man es auch in Lehrveranstaltungen schafft, eine kritische Masse zu erreichen. Dies würde vor allem auch dem didaktischen Konzept der Handlungsorientierung sowie des kollaborativen Lernens entsprechen, die den Lernenden möglichst aktiv in die Lehre einbinden wollen und durch die aktive Beschäftigung mit den Lerninhalten einen höheren Kompetenzzuwachs erzielen sollen, als es klassische Lehrkonzepte vermögen und die als geeignet zu bezeichnen sind, die Anforderungen des Bologna-Prozesses erfüllen zu können (Widulle 2009).

\subsection{Die gruppenspezifische kritische Masse und ihre Bedeutung für die Lehre}

Befasst man sich mit der Frage, wie bei neuen kritische Masse-Systemen die ersten Nutzer gewonnen werden können und die Nutzungsintensität so gesteigert werden kann, dass eine kritische Masse erreichbar wird, bietet der Ansatz der gruppenspezifischen kritischen Masse einen weiteren Erklärungsansatz. Demnach wird die Adoptionsentscheidung eines einzelnen Nachfragers grundlegend dadurch determiniert, dass er in seinem relevanten Milieu, also der für ihn relevanten sozialen Gruppen, eine zufriedenstellende Menge an Teilnehmern perzipiert oder in naher Zukunft mit dieser Menge an Teilnehmern rechnet (Weiber 1995, S. 54-57). Der Ansatz der gruppenspezifischen kritischen Masse geht weiter davon aus, dass ein einzelner Nachfrager besonders dann zur Adoption eines kritische Masse-Systems bereit sein wird, wenn eine ausreichende Zahl an Personen aus diesem relevanten Milieu bereits vor ihm adoptiert haben (Rogers und Kincaid 1981, S. 258 ff.). Die handelnden Personen innerhalb dieser Milieus kennzeichnen sich durch ähnliche Kommunikations- und Interaktionsbedürfnisse, sodass davon ausgegangen werden kann, dass diese Personen einer homogenen Kommunikationsgruppe zuzuschreiben sind. Auf die kritische Masse im Gesamtsystem bezogen müssen demnach zwei Faktoren berücksichtigt werden. Zum einen muss innerhalb der einzelnen Gruppen eine kritische Masse erreicht werden, die aber aufgrund der Gegebenheiten in den Gruppen leichter zu erreichen ist, als in einem großen heterogenen Gesamtsystem. Zum anderen muss darüber hinaus eine möglichst intensive Interaktion beziehungsweise Kommunikation zwischen den Gruppen angestrebt werden, da „erst durch die Kaskadierung der Nutzung zwischen den Gruppen der Markter- 
folg eines kritische Masse-Systems in der Gesamtpopulation gesichert ist (Weiber 1995, S. 55).“

\subsection{Besonderheiten der kritischen Masse in Lehrveranstaltungen}

Um zu verstehen unter welchen Voraussetzungen eine kritische Masse, etwa bei dem Einsatz eines Wikis in Lehrveranstaltungen, erreicht werden kann, müssen zunächst die besonderen Gegebenheiten der installierten Basis und des damit verbundenen Diffusions- und Adoptionsprozesses näher betrachtet werden.

Eine Besonderheit der installierten Basis von Lehrveranstaltungen besteht darin, dass sie den teilnehmenden Lernenden entspricht. Im Gegensatz zu kritische Masse-Systemen im Allgemeinen ist die installierte Basis eine feste, gegebene GröBe und ändert sich im Verlauf der Veranstaltung nicht. Daher kann in Lehrveranstaltungen in der Regel eine kritische Masse nicht durch das Erreichen einer bestimmten Nutzerzahl zustande kommen. ${ }^{1}$ Hier bietet die bereits erläuterte Nutzungsintensität (vgl. 2.2), welche den zentralen Nutzen stiftenden Faktor in kritische Masse-Systemen darstellt, den entscheidenden Erklärungsbeitrag.

Bezogen auf den Einsatz von Web 2.0-Anwendungen in Lehrveranstaltungen, ist darüber hinaus noch eine weitere Besonderheit zu beachten, die bei der Erreichung einer kritischen Masse eine wichtige Rolle spielt. So kann die Adoption durch die Aufgabenstellung ,erzwungen“ werden, was gezielt zur Initiierung der Nutzungsprozesse, z.B. in einem Wiki, genutzt werden kann. Hier ist allerdings zu beachten, dass die Aufgabenstellung die Nutzungsprozesse gezielt fördern sollte, um einen Anstieg der Nutzungsintensität bis zur Erreichung einer kritischen Masse zu gewährleisten. Im Hinblick auf die Erreichung der gruppenspezifischen kritischen Masse sollte dies zusätzlich sowohl innerhalb der Gruppe als auch zwischen den einzelnen Gruppen gelingen. Zur Umsetzung dieser Anforderungen eignen sich Aufgabenstellungen, die möglichst offen formuliert werden und so den Gruppen die Möglichkeit lassen, selbstständig individuelle Ergebnisse zu erarbeiten (Niegemann et al. 2008, S. 322).

Eine weitere Besonderheit ist darin zu sehen, dass die Adoptionsdauer in Lehrveranstaltungen durch die Dauer des Semesters vorgegeben ist. So endet die Adoption des Systems in der Regel mit Ende der Lehrveranstaltung. Allerdings können die Handlungsprodukte der Studierenden weiter genutzt werden, z.B. um ein Wiki in zukünftigen Lehrveranstaltungen weiter auszubauen und die bereits vorhandenen Inhalte zu überarbeiten.

\footnotetext{
1 Eine Ausnahme dazu bestünde, wenn die Web 2.0-Anwendung optional von den Studierenden eingesetzt werden kann und nicht vorgegebener Pflicht-Bestandteil der Aufgabenstellung ist.
} 


\section{$3 \quad$ Konkretisierendes Beispiel einer Blended-Learning Veranstaltung}

\subsection{Struktur der Lehrveranstaltung}

Im Folgenden soll als konkretisierendes Beispiel ein Blended Learning Szenario aus dem Sommersemester 2009 (5 ECTS-Punkte) zum Thema E-Business, das als eine von den Studierenden wählbare Option im Rahmen einer ökonomischen Veranstaltungsreihe an einer deutschen Universität angeboten wird, vorgestellt werden.

Die Konzeption der betrachteten Lehrveranstaltung orientiert sich an dem Lernszenario „Lernaktive Vorlesung“ nach Gersch und Weber (2007, S. 24f.), in dem die klassischen Vorlesungen durch eine offenere Plenumsform ersetzt werden, in denen der Dozent einzelne thematische Inputs und inhaltliche Grundlagen im Dialog mit den Studierenden erarbeitet. Diese Grundlagen dienen dazu, anschließend in selbst gesteuerten Arbeitsgruppen von fünf bis sechs Studierenden eine konkrete projektorientierte Gruppenarbeit durchzuführen, in der sich die Studierenden intensiv und selbstverantwortlich mit den Inhalten der Plenumsveranstaltungen auseinandersetzen können (vgl. Abbildung 1).

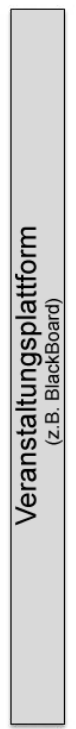

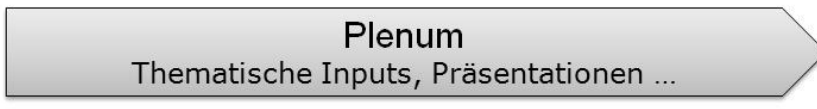

Angeleitete Gruppen

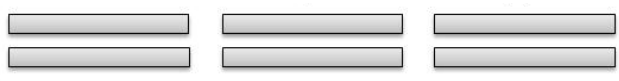

Gruppenstärke ca. 30 Teilnehmer

Professor

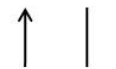

Beratung

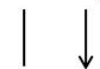

Mitarbeiter

und Tutoren

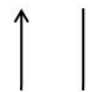

Beratung

Selbst gesteuerte Arbeitsgruppen
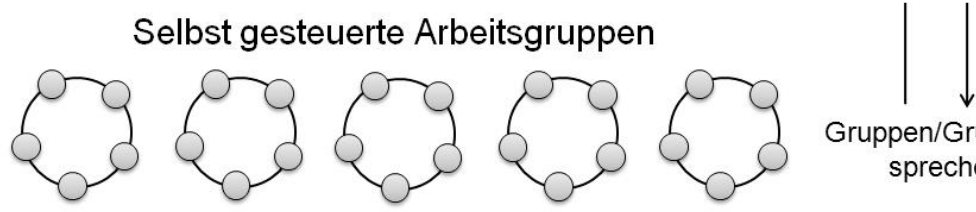

Gruppen/Gruppensprecher

\section{Abbildung 1: Veranstaltungsstruktur (vg1. Sloane 2008, S. 7)}

Die Studierenden erstellen Wiki-Beiträge, in denen sie die Inhalte der Plenumssitzungen anhand von Aufgabenstellungen aufbereiten. Ziel ist es, veranstaltungsbegleitend ein durch die Studierenden selbst erstelltes digitales Veranstaltungsskript in Form eines Wikis zu entwickeln, welches die Studierenden als Grundlage für die 
Vorbereitung auf die abschließende Klausur nutzen können. Der konkrete Einsatzzweck des Handlungsproduktes der Lernenden kann dabei zusätzliche Anreize zur Steigerung der intrinsischen Motivation schaffen. Dafür wird jeder Arbeitsgruppe ein Plenumsthema zugewiesen, für dessen Aufbereitung die jeweilige Gruppe verantwortlich zeichnet. Neben der Aufbereitung dieses Themas haben die Studierenden zusätzlich die Aufgabe, Beurteilungen im Hinblick auf die Eignung der Wiki-Beiträge der anderen Arbeitsgruppen zur Erreichung der Zielsetzung, nämlich der Klausurvorbereitung, zu formulieren. Diese dienen den verantwortlichen Arbeitsgruppen als wichtiges Feedback, um ihre Wiki-Beiträge zu optimieren und fördern zusätzlich die kollaborative Zusammenarbeit zwischen den Studierenden.

\subsection{Ergebnisse der Lehrveranstaltung}

Die Evaluationsergebnisse und die entstandenen Wiki-Beiträge der Studierenden lassen darauf schließen, dass in der betrachteten Lehrveranstaltung gruppenspezifische kritische Massen in den einzelnen Arbeitsgruppen erreicht werden konnten und das hieraus eine direkt messbare Verbesserung der Lernzufriedenheit und des Lernerfolges erzielt wurde.

Einen ersten Hinweis auf die Erreichung einer kritischen Masse kann die Erhebung der Aktivitäten im Veranstaltungswiki geben. So erstellten 35 Studierende, die in sechs Arbeitsgruppen eingeteilt waren, 168 Wiki-Seiten mit insgesamt 73491 Wörtern. Das entspricht im arithmetischen Mittel 2100 Wörtern pro Studierendem und 12249 Wörtern pro Arbeitsgruppe. Für die Erstellung der Endergebnisse benötigten die Studierenden dabei 3308 Überarbeitungsschritte, was im Mittel 95 Bearbeitungsschritten pro Studierendem und 551 Arbeitsschritten pro Arbeitsgruppe entspricht.

Anzumerken ist, dass von den Betreuern der Veranstaltung ein Arbeitspensum von ca. 2500 Wörtern pro Arbeitsgruppe als Minimalziel vorgegeben war. Dieses Arbeitspensum wurde bei Weitem überschritten, was ein weiteres Indiz dafür ist, dass die Veranstaltungskonzeption zu einer hohen Motivation und dadurch zu einer hohen Nutzungsintensität des Wikis aufseiten der Studierenden geführt hat, worin ein weiterer Anhaltspunkt für das Erreichen einer kritischen Masse gesehen werden kann.

Neben dieser rein quantitativen Erhebung der Wiki-Ergebnisse ist zu bemerken, dass auch die Qualität der erstellten Beiträge als durchweg hoch zu bewerten ist. Die von den Studierenden erstellten Beiträge wurden durch umfassende Quellenrecherche belegt und sowohl die erstellten Texte als auch Grafiken eignen sich gut, um den Kommilitonen eine umfassende Klausurvorbereitung zu ermöglichen. Dies führt zu einem weiteren Vorteil des Einsatzes eines solchen Wiki-Konzeptes, der darin zu sehen ist, dass die erstellten Inhalte auch für die Nutzung in anderen und zukünftigen Lehrveranstaltungen geeignet sind und nur geringer redaktioneller Nachbearbeitung durch die Betreuer bedürfen. Die sich hier abzeichnende hohe 
Motivation, die anhand der Nutzungsintensität, die weit über dem geforderten Niveau lag, belegt werden kann, führte nicht zuletzt auch zu direkt messbaren Erfolgen hinsichtlich Lernzufriedenheit und Lernerfolg.

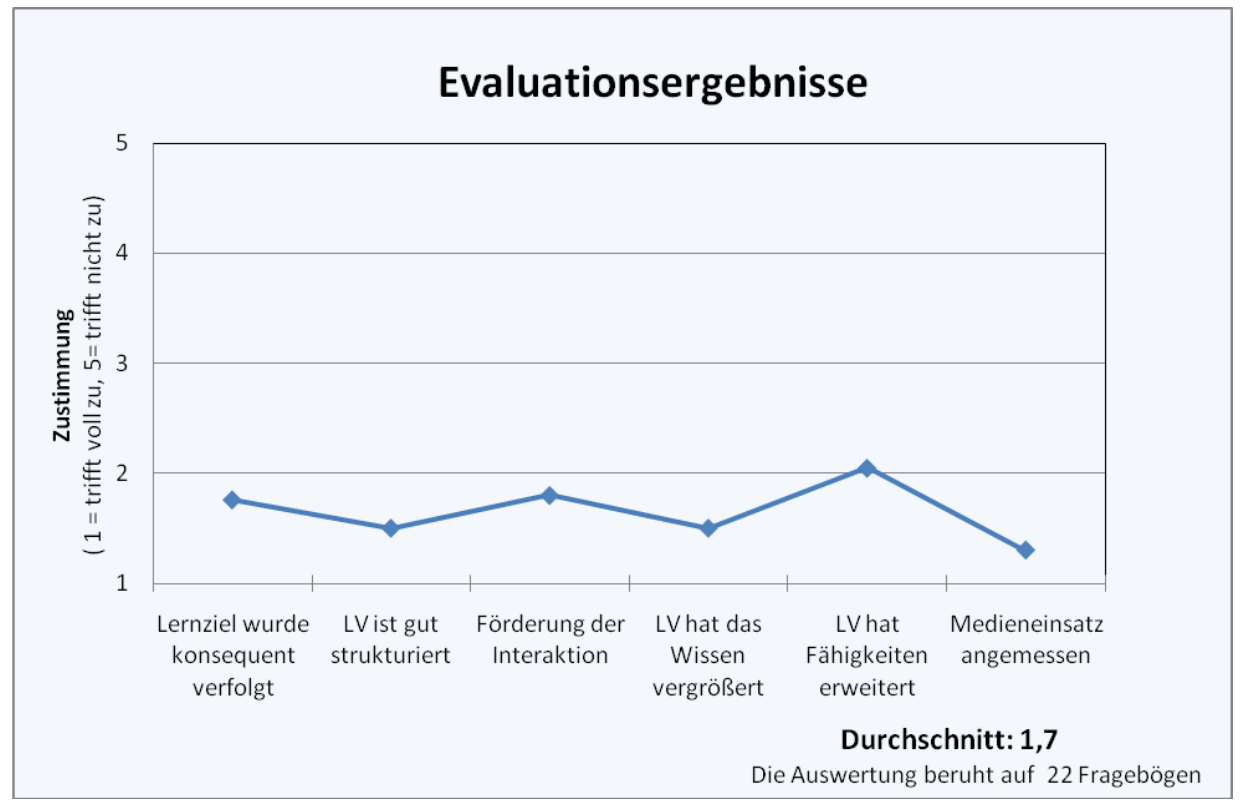

Abbildung 2: Evaluationsergebnisse der betrachteten Lehrveranstaltung

Abbildung 2 zeigt einen Ausschnitt der im Evaluationsbogen abgefragten Items. Die durchweg positive Zustimmung der teilnehmenden Studierenden weist auf eine hohe Lernzufriedenheit hin, die nicht zuletzt auf den Einsatz des Wikis und der Erreichung der kritischen Masse in dieser Veranstaltung zurückzuführen sind. Auch der Lernerfolg kann mit einem erreichten Notendurchschnitt von insgesamt 1,5 für die Gruppenarbeit und 2,1 für die gesamte Veranstaltung als hoch eingestuft werden.

\section{$4 \quad$ Fazit und Ausblick}

Wie auch anhand des betrachteten Beispiels gezeigt werden kann, bietet der sinnvolle, also in die Veranstaltungskonzeption integrierte Einsatz von Web 2.0Anwendungen, hier in Form eines Wikis, ein hohes Potential für einen Mehrwert in der Hochschullehre. Voraussetzung dafür ist jedoch ein konzeptioneller Wandel der Lehrveranstaltungen vom klassischen Frontalformat hin zu handlungsorientierten, kollaborativen Veranstaltungskonzeptionen, die den Lernenden einen möglichst hohen Spielraum für selbst gesteuertes Arbeiten ermöglichen. 
Werden die Anreize in Form möglichst offen gehaltener Aufgabenstellungen, mit vorgegeben Zielen in Form konkreter Handlungsprodukte gesetzt, lässt sich mit Hilfe der angesprochenen Web 2.0-Anwendungen eine kritische Masse erreichen, deren Überschreiten zu einer deutlich intensiveren Beschäftigung der Lernenden mit den Lehrinhalten führen kann. Diese intensive Beschäftigung mit den Lehrinhalten zum einen und die Auseinandersetzung mit modernen, digitalen Kommunikations- und Kooperationswerkzeugen zum anderen, können somit eine mögliche Antwort auf die neuen Anforderungen der Hochschullehre sein, die nicht zuletzt durch die im Bologna-Prozess eingeleiteten Veränderungen getrieben sind.

In den kommenden Semestern wird die Konzeption der betrachteten Lehrveranstaltung weiter zu entwickeln sein. So sollen die Aufgabenstellungen und die Konzeption der Gruppenarbeitsphase im Hinblick auf die Erreichung einer kritischen Masse weiter verbessert und die Interaktionsprozesse zwischen den einzelnen Gruppen noch stärker gefördert werden. Dadurch kann eine noch intensivere Auseinandersetzung mit den Lerninhalten zwischen den Studierenden gefördert werden. Darüber hinaus endet bislang mit der Veranstaltung auch nach jedem Semester die Nutzung des Wikis. Das mögliche Potenzial der erstellten Inhalte bleibt somit ungenutzt. Hier ist geplant, das Wiki und die darin erstellten Inhalte in zukünftigen Veranstaltungen einzusetzen, wodurch die Inhalte kontinuierlich erweitert, vertieft und die Informationsbasis für die Studierenden vergrößert wird.

Nicht zuletzt soll ein geeignetes Evaluationsverfahren, mit dem die hier anhand von ersten Erhebungen und quantitativen Indizien getroffenen Annahmen nachhaltiger überprüft werden können, entwickelt werden. Um den Diffusionsverlauf und damit die Erreichung einer kritischen Masse nachvollziehen zu können, ist eine Betrachtung des Nutzungsverhaltens der Studierenden im Zeitablauf erforderlich. Hier existieren bereits erste Bestrebungen mithilfe geeigneter Netzwerkanalysetools eine differenziertere Betrachtung des Nutzungsverlaufs und damit der Erreichung der kritischen Masse in den Veranstaltungswikis zu ermöglichen. Dies soll ein noch besseres Verständnis der Kooperations- und Kollaborationsprozesse in Lernarrangements mit kritischem Masse-Charakter und damit eine weitere Optimierung des Veranstaltungskonzepts ermöglichen.

\section{Literatur}

BMBF (2009) Der Bolognaprozess. http://www.bmbf.de/de/3336.php. Abruf am 08.09.2009

BMBF, Klieme E (2007) Zur Entwicklung nationaler Bildungsstandards eine Expertise. http://www.bmbf.de/pub/zur_entwicklung_nationaler_bildungssta ndards.pdf. Abruf am 09.09.2009.

Clements MT (2004) Direct and indirect network effects: are they equivalent?. IJIO 22(5): 633-645. 
Gersch M, Weber P (2007) Serviceplattformstrategien für E-Learning Geschäftsmödelle. Zeitschrift für E-Learning - Lernkultur und Bildungstechnologie, 2. Jg., Heft 3, S.19-28.

Gudjons H (2008) Handlungsorientiert lehren und lernen: Schüleraktivierung, Selbsttätigkeit, Projektarbeit. Klinkhardt, Bad Heilbrunn.

Katz M, Shapiro C (1994) Systems Competition and Network Effects. JEP 8(2): 93-115.

Knorr E (2003) 2004: The Year of Web Services.

http://www.cio.com/article/32050/2004_The_Year_of_Web_Services. Abruf am 16.11.2009.

Kroeber-Riel W, Weinberg P, Gröppel-Klein A (2009) Konsumentenverhalten. Vahlen, München.

Liehr M (2005) Die Adoption von Kritische-Masse-Systemen : das Problem der individuellen kritischen Masse. Dt. Univ.-Verl, Wiesbaden.

Lim B-L, Choi M, Park M-C (2003) The late take-off phenomenon in the diffusion of telecommunication services: network effect and the critical mass. IEP 15(4): 537-557.

Niegemann HM, Domagk S, Hessel S, Hein A, Hupfer M, Zobel A (2008) Kompendium multimediales Lernen. Springer, Heidelberg.

O'Reilly T (2005) What is Web 2.0?. http://oreilly.com/web2/archive/what-isweb-20.html. Abruf am 08.09.2009.

O’Reilly T (2006) Web 2.0 Compact Definition: Trying Again. http:/ / radar.oreilly.com/2006/12/web-20-compact-definition-tryi.html. Abruf am 08.09.2009.

Rogers EM (1990) The „Critical Mass“ in the Diffusion of Interactive

Technologies. Modelling the Innovation. In: Carnevale M, Lucertini M, Nicosia S (Hrsg) Communications, Automation and Information Systems. Elsevier, Amsterdam.

Rogers EM (2003) Diffusion of innovations. Free Press, New York.

Rogers EM, Kincaid DL (1981) Communication networks toward a new paradigm for research. Free Press, New York.

Sloane PFE (2008) Kompetenzentwicklung in universitären Massenveranstaltungen? - Lerntheoretische Überlegungen und didaktische Konsequenzen. http://pbwi2www.uni-paderborn.de/WWW/VH B/VHB-Online.nsf/id/DE_Bericht_zur_Arbeitstagung_Massenveranstaltung en_in_der_betriebswirtschaftlichen_Ausbildung_Probl/\$file/04_Sloane.pdf. Abruf am 08.09.2009. 
Swann GMP (2002) The functional form of network effects. IEP 14(3): 417-429.

Weiber R (1992) Diffusion von Telekommunikation - Problem der Kritischen Masse. Gabler, Wiesbaden.

Weiber R (1995) Systemgüter und klassische Diffusionstheorie - Elemente einer Diffusionstheorie für Kritische Masse-Systeme. In: Stoetzer M-W, Mahler A (Hrsg) Die Diffusion von Innovationen in der Telekommunikation. Springer, Berlin.

Weinert FE (2001) Vergleichende Leistungsmessung in Schulen - eine umstrittene Selbstverständlichkeit. In: Weinert FE (Hrsg) Leistungsmessungen in Schulen. Beltz Verlag, Basel.

Widulle W (2009) Handlungsorientiert lernen im Studium: Arbeitsbuch für sozialpädagogische Berufe. VS Verlag, Wiesbaden.

Wopp C (1986) Stichwort: Unterricht, handlungsorientierter. In: Lenzen D (Hrsg) Enzyklopädie Erziehungswissenschaft: Handbuch und Lexikon der Erziehung (Band 3). Klett-Cotta, Stuttgart. 